\title{
Research defects of fatigue failure of screws in solid works instrumentation
}

\begin{abstract}
An alternative to waste incineration is municipal waste recycling by moulding in extrusion machines to make pellets to be further used in the fuel or construction industries. The profitability of a waste recycling facility is dependent on a sound choice of extrusion equipment with the best value for mone.1-5
\end{abstract}

Keywords: fatigue failure, screw, refuse processing, deflected mode, mode of deformation, recycling facility, non-destructive, industrial objects, cosmos works, raw materials, increased friction, matrix, screw design
Volume 2 Issue 3 - 2018

\author{
Kirill Epifantsev, Mishura T \\ Ass professor, Saint-Petersburg State University of Aerospace \\ Instrumentation, Russia
}

\begin{abstract}
Correspondence: Kirill Epifantsev, Ass professor, SaintPetersburg State University of Aerospace Instrumentation, Russia,Tel+79633437759, Email epifancew@gmail.com
\end{abstract}

Received: March 30, 2018 | Published: June 22, 2018

\section{Introduction}

The development of non-destructive testing in Saint-Petersburg State University of aerospace instrumentation (SUAI) is reflected in the appearance of new terms that are used in practice, scientific papers and technical descriptions. Non-destructive control increasingly contacts with adjacent areas involved in assessing the actual technical condition of the facilities, determining the possibility of their further operation and the terms of safe operation (resource problem). In this paper, the methods of nondestructive testing of industrial objects, as well as various types of this method, such as magnetic, electric, which are used to create new laboratory work in the educational process. In this paper, a finite element method was used to study the auger in the program Cosmos Works (Figure 1). ${ }^{6-10}$

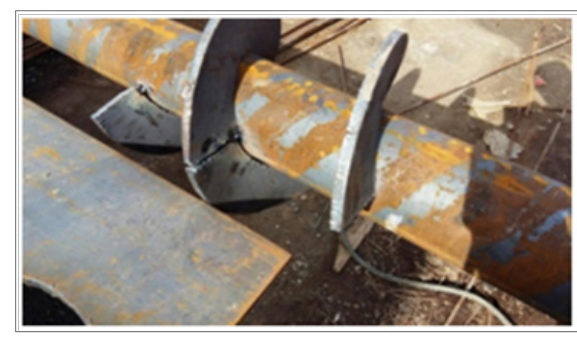

Figure I Screw defects-tears.

\section{Materials and methods}

When modeling extruders for waste processing, much attention is paid to designing and testing the work of the auger, the main working member involved in grinding, heating and preparing for molding a plastic mass. The mass through the matrix (Figure 2). The auger compresses the mass to the accumulation point $\mathrm{m}$. Pp, after which, it becomes possible to create the pressure $\mathrm{p} 1$ necessary to pass Only when there is a balance on both sides of the extruder, the mass can come out of the die dies. However, if the extruder is conventionally divided into 4 work areas, at the moment of increasing pressure $\mathrm{P}$, $\mathrm{MPa}$ and the maximum loading of the screw $\mathrm{p} \mathrm{kg} / \mathrm{m} 3$, a maximum pressure area is created (in Figure 1 zone " 2 "), which is capable of deforming the auger. In an auger machine, an increase in mass consumption requires an increase in the speed of the screw, and a corresponding increase in the loading of the screw by the material. At the same time, the pressure (Figure 1) rises to Pn, because the increased feed rates lead on the same matrix to increased friction in the die dies, which adversely affects also the maximum abrasive area for screw turns. With the auger manufacturing technology - welding the turns to the shaft by welding in $\mathrm{CO}_{2}$, cracks and loosening of the screw turns (Figure 2). Naturally during the operation for forming RDF raw materials, it was assumed that the following load acts on the extruder screw: the torque on the coupling shaft produced by the engine is $5000 \mathrm{~N}$. The temperature of the raw material heating inside the extruder reaches $500{ }^{\circ} \mathrm{C}$. It is necessary to determine the zones with the greatest accumulation of load, displacement, deformation (Figure 2). Thus, creating the parameters that are as close as possible to the real operating conditions, it is possible to detect the most loaded zones of the structure during the research and thus to improve the screw design in the stage of design and selection of materials. ${ }^{11}$

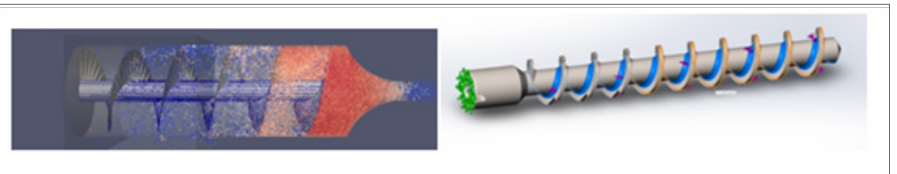

Figure 2 Screwing the auger into a grid using the finite element method, indicating the strength of the action on the screw.

\section{Results and discussions}

The process of molding the biomass is accompanied by a sufficiently high pressure, which is necessary for the destruction of the residual fibers and the compaction of the processed batch. Along with the negative effect of pressure on the turns of the auger, with an unbalanced, unbalanced shape of the screw, the energy spent on molding. The Solid works software allows to simulating methods for diagnostics on the basis of application of virtual measuring instruments with minimal systematic error. This creates ideal conditions for the creation of new technologies in the field of innovation and the development of tools for express diagnostics of most technological processes (Figure 3). ${ }^{12}$ 


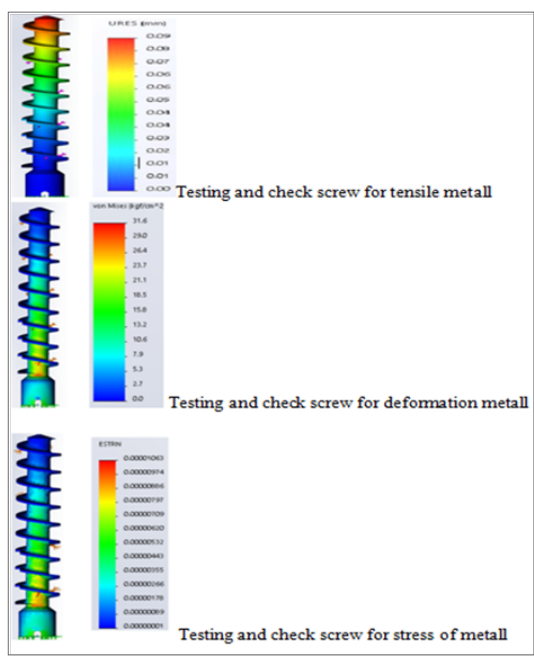

Figure 3 Results of testing screw.

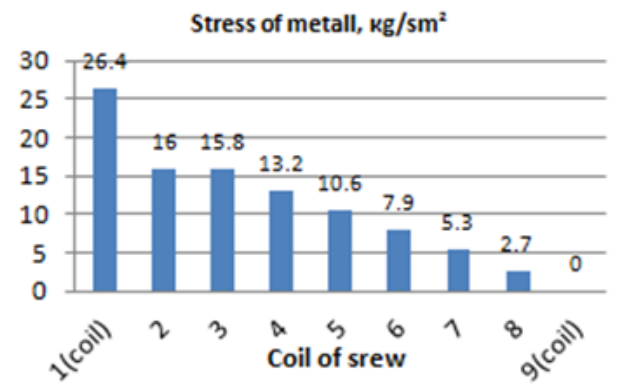

Figure 4 Results of stress.

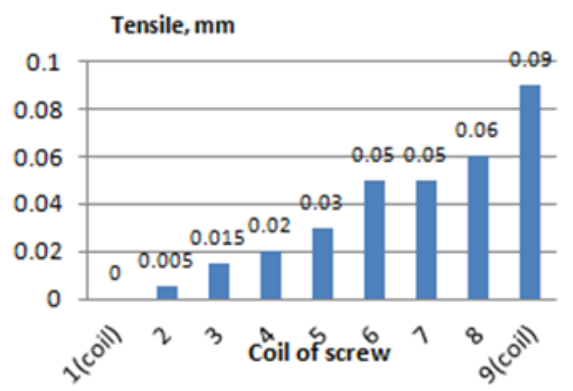

Figure 5 Results of tensile.

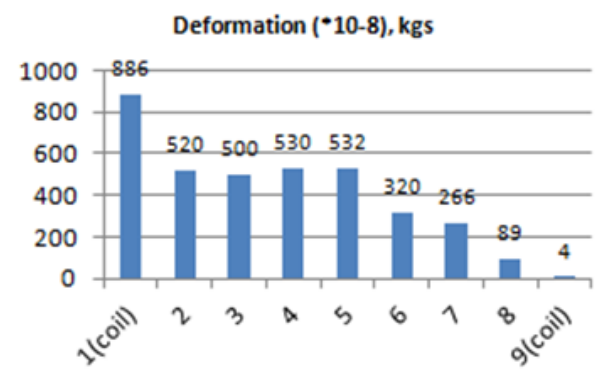

Figure 6 Results of Deformation screw.

\section{Summary}

In Fig 4 -6 will be see results of experiment. Thus the software allowed to identify the most dangerous areas in the zone of bright red colors, which can become a zone of fatigue destruction of the screw and bring to the blocking of the machine (Figures 4-6). In addition, these parts are subject to the most wear and the process of highly abrasive action, which will cause a decrease in pressure inside the machine body, and thus deterioration in the quality of the molded pellets. The stress and strain are inversely proportional to the displacement, which proves the maximum coefficient of pressure on the screws in this region. Therefore, the 7 th and 8 th turns must be welded together with the additional reinforcement rib in order to compensate the stresses.

\section{Conclusion}

The software allows to simulate methods for diagnostics on the basis of application of virtual measuring instruments with minimal systematic error. This creates ideal conditions for the creation of new technologies in the field of innovation and the development of tools for express diagnostics of most technological processes

\section{Acknowledgments}

The results of this work were marked on the grant for young scientists (Government of St. Petersburg, 2017), Scholarship of the President SP-2381.2018.1.

\section{Conflict of interest}

The author declares there is no conflict of interest.

\section{References}

1. Qingqing, H. Recent trends in rock dust modifications for improved dispersion and coal dust explosion mitigation. Journal of Loss Prevention in the Process Industries. 2016;41:121-128.

2. Qingqing H. Optimized reagent dosage effect on rock dust to enhance rock dust dispersion and explosion mitigation in underground coal mines. Powder Technology. 2016;301:1193-1200.

3. Title 30-Mineral Resources. Vol. 1, parts 1-199. Washington: U.S. Government Printing Office, 2009. 767 p.

4. Title 30-Mineral Resources. Vol. 2, parts 600-699. Washington: U.S. Government Printing Office, 2009. 750 p.

5. Title 30-Mineral Resources. Vol. 3, parts 700 to end. Washington: U.S. Government Printing Office, 2009. 750 p.

6. Order No. 440 dated 19 November 2013 on Approval of the Coal Mine Safety Rules Federal Industrial Safety Rules and Regulations. Federal Service for Ecological, Technological, and Nuclear Supervision.

7. Lindenau NI. Origin, Prevention, and Suppression of Spontaneous Fires in Coal Mines. 1977. 387 p.

8. Yinlin J, Peter W, Zhijun W. A comparative study of dust control practices in Chinese and Australian longwall coal mines. International Journal of Mining Science and Technology. 2016;26(2):199-208.

9. Lebecki K. Gas in mines. Katowice: The Main Institution Gornictwa, 2004. $399 \mathrm{p}$.

10. European Standard EN 13205. Workplace Environment: Assessment of the Productivity of Air-Borne Particle Concentration Meters. German version. 2016. 
11. European Standard EN 481. Determination of Conventions on a Fraction Part for Suspended Solids Measurement in the Workplace. German version. 1991.
12. International Standard ISO 7708. Air Quality. Determination of Fractions by Particle Size for Hygiene-Related Fractions. 1995. 